\title{
Sequential voting rules and multiple elections paradoxes
}

\author{
Lirong Xia \\ State Key Laboratory of \\ Intelligent Technology and Systems \\ Department of Computer Science \\ and Technology, Tsinghua University \\ Beijing 100084, China \\ xialirong@gmail.com
}

\begin{abstract}
Multiple election paradoxes arise when voting separately on each issue from a set of related issues results in an obviously undesirable outcome. Several authors have argued that a sufficient condition for avoiding multiple election paradoxes is the assumption that voters have separable preferences. We show that this extremely demanding restriction can be relaxed into the much more reasonable one: there exists a linear order $\mathbf{x}_{1}>$ $\ldots>\mathbf{x}_{p}$ on the set of issues such that for each voter, every issue $\mathbf{x}_{i}$ is preferentially independent of $\mathbf{x}_{i+1}, \ldots, \mathbf{x}_{p}$ given $\mathbf{x}_{1}, \ldots, \mathbf{x}_{i-1}$. This leads us to define a family of sequential voting rules, defined as the sequential composition of local voting rules. These rules relate to the setting of conditional preference networks (CP-nets) recently developed in the Artificial Intelligence literature. We study in detail how these sequential rules inherit, or do not inherit, the properties of their local components. We focus on the case of multiple referenda, corresponding to multiple elections with binary issues.
\end{abstract}

\section{Introduction}

In many contexts, a group of voters has to make a common decision on several possibly related issues, such as in multiple referenda, or voting for committees (the issues then are the positions to be filled - see [1]). As soon as voters have preferential dependencies between issues, it is generally a bad idea to decompose a vote problem on $p$ issues into a set of $p$ smaller problems, each one bearing on a single issue: "multiple election paradoxes" (or "paradoxes of multiple referenda") then arise.

Such paradoxes have been studied in several papers, with two slightly different views. In $[6,15]$, voters can vote only $Y$ or $N$ on each issue; the paradox occurs when the set of

\author{
Mingsheng Ying \\ State Key Laboratory of \\ Intelligent Technology and Systems \\ Department of Computer Science \\ and Technology, Tsinghua University \\ Beijing 100084, China \\ yingmsh@mail.tsinghua.edu.cn
}

propositions that win, when votes are aggregated separately for each proposition received the fewest votes when votes are aggregated by combination: for instance, suppose there are 3 propositions $A, B, C$ and three voters voting respectively for $A B \bar{C}, A \bar{B} C$ and $\bar{A} B C$. Propositionwise aggregation leads to $A B C$, which $A B C$ receives support for not a single voter. The paradox studied in [10] is a little bit different. They show that voting issue by issue is feasible (to some extent) when preferences are separable, and that it generally fails when they are not (a voter's preferences are separable if her preferences on an issue does not depend on the choice to be made for other issues). However, separability is an extremely strong assumption that is unlikely to be met in practice. Furthermore, even when preferences are separable, some paradoxes still arise, such as the choice of a Pareto-dominated outcome [13, 2].

Example 1 A common decision has to be made about whether or not to build a new swimming pool $(S$ or $\bar{S})$ and a new tennis court ( $T$ or $\bar{T})$. Assume that the preferences of voters 1 and 2 are $S \bar{T} \succ \bar{S} T \succ \bar{S} \bar{T} \succ S T$, those of voters 3 and 4 are $\bar{S} T \succ S \bar{T} \succ \bar{S} \bar{T} \succ S T$ and those of voter 5 are $S T \succ S \bar{T} \succ \bar{S} T \succ \bar{S} \bar{T}$.

The first problem with Example 1 is that voters 1 to 4 feel ill at ease when asked to report their projected preference on $\{S, \bar{S}\}$ and $\{T, \bar{T}\}$. Only voter 5 knows that whatever the other voters' preferences about $\{S, \bar{S}\}$ (resp. $\{T, \bar{T}\}$ ), she can vote fot $T$ (resp. $S$ ) without any risk of experiencing regret (this is called simple voting in [1]). The analysis of the paradox in [10] considers that voters report their preferences optimistically (thus voters 1-2 report a preference for $S$ over $\bar{S}$ ), but this assumption, even if it has been justified by experimental studies (see [14]), remains arbitrary, and would not necessarily carry on to more complex situations such as a voter with the following preference relation: $A B C \succ \bar{A} \bar{B} \bar{C} \succ \bar{A} \bar{B} C \succ \bar{A} B \bar{C} \succ \bar{A} B C \succ A \bar{B} \bar{C} \succ A \bar{B} C \succ A B \bar{C}:$ only a very optimistic voter would report a preference for $A$ (except, of course, if some prior beliefs about the others' preferences make him believe that the common decision about $B$ and $C$ will be $B C$.)

The second problem (the paradox itself) is that under this 
assumption that voters report optimistic preferences, the outcome in Example 1 will be $S T$, which is the worst outcome for all but one voter, and a fortiori, is a Condorcet loser. Lacy and Niou [10] and Benoit and Kornhauser [2] give other examples, with three issues, leading to an even worse paradox where the outcome is ranked last by everyone.

The main question is now, how can these paradoxes be avoided? Reformulating the question in a more constructive way, how should a vote on related issues be conducted? We argue that we have to choose one of the following two ways, each of which has some specific pitfalls: either work at the global level and vote for combinations of values, or work at the local level and vote separately on each issue, sequentially or simultaneously ${ }^{1}$.

The "global way" consists in giving up decomposing the global vote into local votes and voting for combinations of values. This solution is supported by Brams et al. [5, 6]. There is some ambiguity on how the process should be conducted, thus leading to three possible methods:

1. ask voters to report their entire preference relation on the set of alternatives, and then apply an usual voting rule such as Borda.

2. ask voters to report only a small part of their preference relation and apply a voting rule that needs this information only, such as plurality;

3. limit the number of possible combinations that voters may vote for.

From a theoretical point of view, Solution 1 works: each agent specifies his preference relation in extenso and then any fixed voting rule is applied to the obtained profile, with no risk of a paradoxical outcome. However, as noticed in [5], this solution is practically unfeasible if the number of issues is more than a small number (say, 3): the exponential number of alternatives makes it unreasonable to ask voters to rank all alternatives explicitly. In other words, implementing such a voting rule on a multi-issue domain needs an exponential protocol. Clearly, exponentially long protocols are not acceptable. Therefore, as soon as the number of issues is not very small, this solution is ruled out by communication complexity considerations.

Solution 2 requires little communication, but it is its only merit. Voting rules that are implementable by a cheap protocol make use of a very small part of the voters' preferences: if the protocol is required to have a polynomial communication complexity, then the voting rule it implements use at most a logarithmic part of the profile. Such rules do exist: not only plurality and veto, but more generally all

\footnotetext{
${ }^{1}$ In the context of assembly elections, these two families of voting rules are called assembly-based and seat-based, respectively $[1,2]$.
}

rules that require, for instance, the $K$ top candidates of each voter, where $K$ is a fixed integer. However, when the number of issues grows, these rules could give extremely bad results. For instance, using plurality when the number of issues is significant and the number of voters small could well result in a situation where no outcome gets more than one vote, in which case plurality would give an extremely poor result.

Solution 3, sketched in [5], presents the chairperson with a very problematic choice. This may be feasible when issues can clearly be packaged into groups of issues such that two groups are clearly independent, but this favorable situation is far from being a general rule.

The "local" way, supported by Lacy and Niou [10] for multiple referenda, consists in sticking to a vote issue by issue, the outcome of the vote on one issue being revealed before the vote on other issues. They show that sequential voting (with whichever agenda) allows for escaping the worst versions of the multiple election paradoxes, namely, it avoids a Condorcet loser to be elected. However, this method still has three major drawbacks. First, the voters may still feel ill at ease when reporting their preference on an issue, when this preference depends on the value of issues not decided yet. Second, the study is based on the assumption that voters will behave optimistically, by reporting the projection of their preferred outcome, which is debatable except in some specific cases. Third, even if a sequential vote avoids the final outcome to be a Condorcet loser, the paradox remains to a large extent, as can be seen on the following example:

Example 2 We have three issues $A, B, C$ and $2 M+1$ voters.

$$
\begin{array}{ll}
\text { M voters: } & A B \bar{C} \succ \bar{A} \bar{B} \bar{C} \succ \ldots \succ A \bar{B} C \succ A B C \\
M \text { voters: } & A \bar{B} C \succ \bar{A} \bar{B} \bar{C} \succ \ldots \succ A B C \succ A B \bar{C} \\
1 \text { voter: } & \bar{A} B C \succ \bar{A} \bar{B} \bar{C} \succ \bar{A} B \bar{C} \succ \bar{A} \bar{B} C \succ A B C \\
& \succ A B \bar{C} \succ A \bar{B} \bar{C} \succ A \bar{B} C
\end{array}
$$

In Example 2, having voters decide first on $A$, then to $B$ and then to $C$, and assuming they behave optimistically, will lead to $A B C$, which is (a) a "nearly-Condorcet loser" (it is Condorcet-dominated by all candidates except one) and (b) Pareto-dominated by half of the outcomes. (More acute paradoxes can be found, but they need more issues and thus more space.) Actually, the reason why the sequential process avoids a Condorcet loser to be elected is only because the last vote is made with a full knowledge of the values of other issues, thus this result loses his significance when the number of issues becomes bigger.

There is a well-known restriction on voter preferences that allows for such paradoxes to be avoided, that is, when all voters have separable preferences across the outcomes of the issues. Then, a voter's preferences on the values of an issue is independent from the values of other issues, and the elicitation process can be performed safely issue by issue 
(and even without needing to resort to sequentiality). Under the separability assumption, voting separately on each issue (either sequentially or simultaneously) enjoys good properties, including the election of a Condorcet winners when there is one. However, the separability restriction is very demanding, and unlikely to be met in practice, especially because separable preferences constitute a very tiny proportion of possible preferences on multiple issues (see [8]).

The question is now, can this extreme separability assumption be relaxed without hampering the nice properties of sequential voting? As it stands, the answer is positive, as the method can be safely applied to far many profiles than separable profiles. Unformally, the condition should be that each time a voter is asked to report his preferences on a single issues or a small set of isssues, these preferences do not depend on the values of the issues that have not been decided yet.

Formally, this can be expressed as the following condition: there is a linear order $O=\mathbf{x}_{1}>\ldots>\mathbf{x}_{p}$ on the set of issues such that for every voter $v$ and every $j$, the preferences of $v$ on $\mathbf{x}_{j}$ are preferentially independent from $\mathbf{x}_{j+1}, \ldots, \mathbf{x}_{p}$ given $\mathbf{x}_{1}, \ldots, \mathbf{x}_{j-1}$. If this property is satisfied, then a simple protocol can be implemented: the voters' preferences about issue $\mathbf{x}_{1}$ are elicited; then a voting rule is applied so as to make a decision on the value of $\mathbf{x}_{1}$; then this chosen value of $\mathbf{x}_{1}$ is communicated to the voters, who then report their preferences on the values of $\mathbf{x}_{2}$ given the fixed value of $\mathbf{x}_{1}$, and so on. Such preference profiles are called $O$-legal and abbreviated as legal for $O=\mathbf{x}_{1}>\ldots>\mathbf{x}_{p}$ in this paper. This protocol generalizes to clusters of issues $I_{1}, \ldots, I_{n}$ where for each voter and each $i, I_{i}$ is preferentially independent of $I_{j+1}, \ldots, I_{m}$ given $I_{1}, \ldots, I_{i-1}$, where $\left\{I_{1}, \ldots, I_{m}\right\}$ forms a partition of the set $I$ of issues.

This domain restriction (O-legality) and the resulting sequential voting rules and correspondences that are then applicable are defined in Section 3. In Section 4 we study in detail the properties of these sequential composition by relating them to the corresponding properties of local voting rules to those of its components. It turns out that while many properties expectedly transfer from local rules to their sequential composition, this is not the case for two important properties, namely neutrality and consensus. In Section 5 we focus on the particular case of multiple referenda, obtained where all issues are binary. In Section 6 we briefly mention further issues. Because of space limit, proofs are omitted ${ }^{2}$.

\footnotetext{
${ }^{2}$ They can be found in a longer version of the paper, at http://www.irit.fr/recherches/RPDMP/persos/JeromeLang/papers/mep-long.pdf.
}

\section{Preferences on multi-issue domains}

Let $I=\left\{\mathbf{x}_{1}, \ldots, \mathbf{x}_{p}\right\}$ be a set of issues. For each $\mathbf{x}_{i} \in I$, $D_{i}$ is the finite value domain of $\mathbf{x}_{i}$. An issue $\mathbf{x}_{i}$ is binary if $D_{i}=\left\{x_{i}, \overline{x_{i}}\right\}$, or equivalently $\left\{1_{i}, 0_{i}\right\}$. (Note the difference between the issue $\mathbf{x}_{i}$ and the value $x_{i}$.) If $X=$ $\left\{\mathbf{x}_{i_{1}}, \ldots, \mathbf{x}_{i_{m}}\right\} \subseteq I$, with $i_{1}<\ldots<i_{p}$, then $D_{X}$ denotes $D_{i_{1}} \times \ldots \times D_{i_{m}} . \times=D_{1} \times \ldots \times D_{p}$ is the set of all alternatives (or candidates). Elements of $x$ are denoted by $\vec{x}, \vec{x}^{\prime}$ etc. and represented by concatenating the values of the issues: for instance, if $I=\left\{\mathbf{x}_{1}, \mathbf{x}_{2}, \mathbf{x}_{3}\right\}, x_{1} \overline{x_{2}} x_{3}$ assigns $x_{1}$ to $\mathbf{x}_{1}, \overline{x_{2}}$ to $\mathbf{x}_{2}$ and $x_{3}$ to $\mathbf{x}_{3}$. We allow concatenations of vectors of values: for instance, let $I=\left\{\mathbf{x}_{1}, \mathbf{x}_{2}, \mathbf{x}_{3}, \mathbf{x}_{4}, \mathbf{x}_{5}\right\}$, $Y=\left\{\mathbf{x}_{1}, \mathbf{x}_{2}\right\}, Z=\left\{\mathbf{x}_{3}, \mathbf{x}_{4}\right\}, \vec{y}=x_{1} \overline{x_{2}}, \vec{z}=\overline{x_{3}} x_{4}$, then $\vec{y} \cdot \vec{z} \cdot \overline{x_{5}}$ denotes the alternative $x_{1} \overline{x_{2} x_{3}} x_{4} \overline{x_{5}}$.

A preference relation on $x$ is a strict order (an irreflexive, asymmetric and transitive binary relation). A linear preference relation $V$ is a complete strict order, i.e., for any $\vec{x}$ and $\vec{y} \neq \vec{x}$, either $\vec{x} \succ \vec{y}$ or $\vec{y} \succ \vec{x}$ holds. We generally note $\vec{x} \succ_{V} \vec{x}^{\prime}$ instead of $V\left(\vec{x}, \vec{x}^{\prime}\right)$.

Let $\{X, Y, Z\}$ be a partition of the set $I$ and $\succ$ a linear preference relation over $X=D_{I} . X$ is (conditionally) preferentially independent of $Y$ given $Z$ (w.r.t. $\succ$ ) if and only if for all $\vec{x}_{1}, \vec{x}_{2} \in D_{X}, \vec{y}_{1}, \vec{y}_{2} \in D_{Y}, \vec{z} \in D_{Z}$,

$$
\vec{x}_{1} \cdot \vec{y}_{1} \cdot \vec{z} \succ \vec{x}_{2} \cdot \vec{y}_{1} \cdot \vec{z} \text { iff } \vec{x}_{1} \cdot \vec{y}_{2} \cdot \vec{z} \succ \vec{x}_{2} \cdot \vec{y}_{2} \cdot \vec{z}
$$

Conditional preferential independence originates in the literature of multiattribute decision theory [9]. Unlike probabilistic independence, it is a directed notion: $X$ may be independent of $Y$ given $Z$ without $Y$ being independent of $X$ given $Z$. Note that preferential independence is weaker than utility independence.

Conditional preference networks, or CP-nets, are a language for specifying preferences based on the notion of conditional preferential independence. They allow for eliciting preferences, and for storing them, as economically as possible. Formally, a $C P$-net $\mathcal{N}$ [3] over a set of attributes (or issues) $I$ is a pair consisting of a directed graph $G$ over $I$ and a collection of conditional preference tables $C P T\left(\mathbf{x}_{i}\right)$ for each $x_{i} \in I$. Appendix 1 gives some fairly detailed background on CP-nets.

Let $O=\mathbf{x}_{1}>\ldots>\mathbf{x}_{p}$ be a linear order on $I$. We say that $\succ$ follows $O=\mathbf{x}_{1}>\ldots>\mathbf{x}_{p}$ if for all $i<p, \mathbf{x}_{i}$ is preferentially independent of $\left\{\mathbf{x}_{i+1}, \ldots, \mathbf{x}_{p}\right\}$ given $\left\{\mathbf{x}_{1}, \ldots, \mathbf{x}_{i-1}\right\}$ with respect to $\succ$.

If $\succ$ follows $O$ then the projection of $\succ$ on $\mathbf{x}_{i}$ given $\left(x_{1}, \ldots, x_{i-1}\right) \in D_{1} \times \ldots \times D_{i-1}$, denoted by $\succ^{\mathbf{x}_{i} \mid \mathbf{x}_{1}=x_{1}, \ldots, \mathbf{x}_{i-1}=x_{i-1}}$, is the linear preference relation on $D_{i}$ defined by: for all $x_{i}, x_{i}^{\prime} \in D_{i}, x_{i} \succ^{\mathbf{x}_{i} \mid \mathbf{x}_{1}=x_{1}, \ldots, \mathbf{x}_{i-1}=x_{i-1}}$ $x_{i}^{\prime}$ iff $x_{1} \ldots x_{i-1} x_{i} x_{i+1} \ldots x_{p} \succ x_{1} \ldots x_{i-1} x_{i}^{\prime} x_{i+1} \ldots x_{p}$ holds for all $\left(x_{i+1}, \ldots, x_{p}\right) \in D_{i+1} \times \ldots \times D_{p}$.

Due to the fact that $\succ$ follows $O$ and that $\succ$ is a linear or- 
der, $\succ^{\mathbf{x}_{i} \mid \mathbf{x}_{1}=x_{1}, \ldots, \mathbf{x}_{i-1}=x_{i-1}}$ is a well-defined linear order as well. Note also that if $\succ$ follows both $O=\mathbf{x}_{1}>\ldots>\mathbf{x}_{p}$ and $O^{\prime}=\mathbf{x}_{\sigma(1)}>\ldots>\mathbf{x}_{\sigma(k-1)}>\mathbf{x}_{i}\left(=\mathbf{x}_{\sigma(k)}\right)>\ldots>\mathbf{x}_{\sigma(p)}$, then $\succ^{\mathbf{x}_{i} \mid \mathbf{x}_{1}=x_{1}, \ldots, \mathbf{x}_{i-1}=x_{i-1}}$ and $\succ^{\mathbf{x}_{i} \mid \mathbf{x}_{\sigma(1)}=x_{\sigma(1)}, \ldots, \mathbf{x}_{\sigma(k-1)}=x_{\sigma(k-1)}}$ coincide. In other words, the local preference relation on $\mathbf{x}_{i}$ depends only on the values of the issues that precede $\mathbf{x}_{i}$ in $O$ and in $O^{\prime}$.

Let $G$ be an acyclic directed graph (DAG) on $I$. A preference relation $\succ$ is compatible with $G$, denoted by $\succ \sim G$, if $\succ$ follows some order $O=\mathbf{x}_{1}>\ldots>\mathbf{x}_{p}$ on $I$ that follows $G$, that is, such that for every edge $\left(\mathbf{x}_{i}, \mathbf{x}_{j}\right)$ in $G$ we have $i<j$. For any two preference relations $\succ_{1}, \succ_{2}$ and CP-net $\mathcal{N}$, we use the following notations: $\succ_{1} \sim \mathcal{N}$ if $\succ_{1}$ extends $\mathcal{N} ; \succ_{1} \sim \succ_{2}$ if there exists a CP-net $\mathcal{N}^{\prime}$ s.t. $\succ_{1} \sim \mathcal{N}^{\prime}$ and $\succ_{2} \sim \mathcal{N}^{\prime} ; \succ_{1} \sim_{\mathcal{N}} \succ_{2}$ if $\succ_{1} \sim \mathcal{N}$ and $\succ_{2} \sim \mathcal{N}$. Lastly, we say $\succ_{1}$ and $\succ_{2}$ are $G$-equivalent, denoted by $\succ_{1} \sim_{G} \succ_{2}$, if and only if $\succ_{1}$ and $\succ_{2}$ are both compatible with $G$ and for any $\mathbf{x} \in V$, for any $\vec{y}, \vec{y}^{\prime} \in \operatorname{Dom}(\operatorname{par}(\mathbf{x}))$ we have $\succ_{1}^{\mathbf{x} \mid \operatorname{par}(\mathbf{x})=\vec{y}}=\succ_{2}^{\mathbf{x} \mid \operatorname{par}(\mathbf{x})=\vec{y}}$. Note that $\succ_{1} \sim_{G} \succ_{2}$ if and only if there exists a CP-net $\mathcal{N}$ whose associated graph is $G$ and such that $\succ_{1}$ and $\succ_{2}$ both extend $\mathcal{N}$. We frequently use the notation $V$ (for "vote") instead of $\succ$.

Example 3 Let $I=\{\mathbf{x}, \mathbf{y}, \mathbf{z}\}$, all three being binary. and let $V$ and $V^{\prime}$ be the following votes:

$$
\begin{aligned}
& V: x y z \succ x y \bar{z} \succ x \bar{y} \bar{z} \succ x \bar{y} z \succ \bar{x} y \bar{z} \succ \bar{x} \bar{y} \bar{z} \succ \bar{x} y z \succ \bar{x} \bar{y} z \\
& V^{\prime}: x y z \succ x y \bar{z} \succ \bar{x} y \bar{z} \succ x \bar{y} \bar{z} \succ \bar{x} y z \succ \bar{x} \bar{y} \bar{z} \succ x \bar{y} z \succ \bar{x} \bar{y} z
\end{aligned}
$$

Let $G$ be the graph over I whose set of edges is $\{(\mathbf{x}, \mathbf{z}),(\mathbf{y}, \mathbf{z})\} . \quad V$ and $V^{\prime}$ are both compatible with $G$. Moreover, $V \sim_{G} V^{\prime}$, since all local preference relations coincide: $x \succ_{V}^{\mathbf{x}} \bar{x}$ and $x \succ_{V^{\prime}}^{\mathbf{x}} \bar{x} ; z \succ_{V}^{\mathbf{z} \mid \mathbf{x}=x, \mathbf{y}=y} \bar{z}$ and $z \succ_{V^{\prime}}^{\mathbf{z} \mid \mathbf{x}=x, \mathbf{y}=y} \bar{z}$; etc. The CP-net that $V$ and $V^{\prime}$ both extend is defined by the following preferences tables: $x \succ \bar{x} ; y \succ \bar{y} ; x y: z \succ \bar{z}$; $x \bar{y}: \bar{z} \succ z ; \bar{x} y: \bar{z} \succ z ; \bar{x} \bar{y}: \bar{z} \succ z$.

\section{Sequential voting rules and correspondences}

We start by recalling briefly some necessary background on voting rules and correspondences (for more details see for instance [4]). Let $\mathcal{A}=\{1, \ldots, N\}$ be a finite set of voters and $X$ a finite set of candidates. A profile w.r.t. $\mathcal{A}$ and $X$ is a collection of $N$ individual linear preference relations over $x$ : $P=\left(V_{1}, \ldots, V_{N}\right)$. Let $P_{\mathcal{A}, x}$ be the set of all preference profiles for $\mathcal{A}$ and $X$. A voting correspondence $C: P_{\mathcal{A}, X} \rightarrow 2^{X} \backslash\{\emptyset\}$ maps each preference profile $P$ of $P_{\mathcal{A}, X}$ into a nonempty subset $C(P)$ of $X$. A voting rule $r: P_{\mathcal{A}, X} \rightarrow \chi$ maps each preference profile $P$ of $P_{\mathcal{A}, X}$ into a single candidate $r(P)$. The correspondence that elects the candidates that are ranked first by the largest number of voters is the plurality correspondence. When there are only two candidates $\{x, y\}$, the majority correspondence maj is defined by $\operatorname{maj}(P)=\{x\}$ (resp. $\{y\}$ if more voters in $P$ prefer $x$ to $y$ (resp. $y$ to $x$ ), and $\operatorname{maj}(P)=\{x, y\}$ in case of tie.

Given a profile $P, x \in X$ is a Condorcet winner (resp. weak Condorcet winner) if it is preferred to any other candidate by a strict (resp. non-strict) majority of voters: for all $y \neq x$, $\#\left\{i: x \succ_{i} y\right\}>\frac{N}{2}$ (resp. $\geq \frac{N}{2}$ ). A Condorcet-consistent rule (resp. correspondence) is a voting rule $r$ (resp. correspondence $C$ ) such that whenever there exists a Condorcet winner $x$ for the profile $P$ then $r(P)=x$ (resp. $C(P)=\{x\})$.

These definitions of voting rules are not concerned with how the votes are elicited from the voters. As in [7] we distinguish between the voting rule and a protocol (which determines which relevant information is elicited, and when, from the voters) that implements it. The deterministic communication complexity of a voting rule $r$ is the worst-case number of bits sent in the best protocol implementing $r$. See [7] for a communication complexity study of various voting rules.

From now on, we assume that the set of candidates is a multi-issue domain $x=D_{1} \times \ldots \times D_{p}$. Sequential voting consists in applying "local" voting rules or correspondences on single issues, one after the other, in such an order that the local vote on a given issue can be performed only when the local votes on all its parents in the graph $G$ have been performed. Note that, unlike in $[5,6,10]$, we do not assume that issues are binary. We now define our crucial domain restriction:

Definition 1 Given a linear order $O=\mathbf{x}_{1}>\ldots>\mathbf{x}_{p}$ on $I$, we define Legal $(O)$ as the set of all profiles $P=$ $\left(V_{1}, \ldots, V_{N}\right)$ such that each $V_{i}$ follows $O$.

We might wonder how strong this restriction is. First of all, note that it is much less demanding than separability. Second, it can be generalized by partitioning the set of issues into subsets $I_{1}, \ldots, I_{q}$ such that $I_{i}$ is preferentially independent of $I_{i+1} \cup \ldots \cup I_{q}$ given $I_{1} \cup \ldots \cup I_{i-1}$. Obviously, all profiles are of this form, the worst case being $q=1^{3}$. However, we can assume without loss of generality (and we will do so in the remainder of the paper) that each cluster consist of a single issue (if this were not the case from the beginning, then each cluster $I_{i}$ can be considered as a new single issue, with domain $D_{I_{i}}=\prod_{\mathbf{x}_{j} \in I_{i}} D_{j}$.)

Definition 2 Let $O=\mathbf{x}_{1}>\ldots>\mathbf{x}_{p}$ be a linear order on $I$, and $\left(r_{1}, \ldots, r_{p}\right)$ a collection of deterministic voting rules (one for each issue $\mathbf{x}_{i}$ ). The sequential voting rule $\operatorname{Seq}\left(r_{1}, \ldots, r_{p}\right)$ is defined on all profiles following $O$ as follows: for any $P=\left(V_{1}, \ldots, V_{N}\right)$ in $\operatorname{Legal}(O)$ :

\footnotetext{
${ }^{3}$ The smaller the size of the subsets, the cheaper the protocol: the communication cost of the protocol for computing a sequential rule using such decomposition into clusters is $\sum_{i=1}^{q} \prod_{\mathbf{x}_{j} \in I_{i}}\left|D_{j}\right|$. The protocol is guaranteed to remain cheap (that is, polynomial) if there exists a constant $K$ (independent from the number of issues and voters) such that $\left|I_{i}\right| \leq K$ for every cluster $I_{i}$.
} 
- $x_{1}^{*}=r_{1}\left(V_{1}^{\mathbf{x}_{1}}, \ldots, V_{N}^{\mathbf{x}_{1}}\right)$;

- $x_{2}^{*}=r_{2}\left(V_{1}^{\mathbf{x}_{2} \mid \mathbf{x}_{1}=x_{1}^{*}}, \ldots, V_{N}^{\mathbf{x}_{2} \mid \mathbf{x}_{1}=x_{1}^{*}}\right)$;

-...

- $x_{p}^{*}=r_{p}\left(V_{1}^{\mathbf{x}_{p} \mid \mathbf{x}_{1}=x_{1}^{*}, \ldots, \mathbf{x}_{p-1}=x_{p-1}^{*}}, \ldots, V_{N}^{\mathbf{x}_{p} \mid \mathbf{x}_{1}=x_{1}^{*}, \ldots, \mathbf{x}_{p-1}=x_{p-1}^{*}}\right)$

Then $\operatorname{Seq}\left(r_{1}, \ldots, r_{p}\right)(P)=\left(x_{1}^{*}, \ldots, x_{p}^{*}\right)$.

Example 4 Let $N=12, I=\{\mathbf{x}, \mathbf{y}\}$ with $D_{\mathbf{x}}=\{x, \bar{x}\}$ and $D_{\mathbf{y}}=\{y, \bar{y}\}$, and $P=\left(V_{1}, \ldots, V_{12}\right)$ the following 12-voter profile:

$$
\begin{array}{ll}
V_{1}, V_{2}, V_{3}, V_{4}: & x y \succ \bar{x} y \succ x \bar{y} \succ \bar{x} \bar{y} \\
V_{5}, V_{6}, V_{7}: & x \bar{y} \succ x y \succ \bar{x} y \succ \bar{x} \bar{y} \\
V_{8}, V_{9}, V_{10}: & \overline{x y} \succ \bar{x} \bar{y} \succ x y \succ x \bar{y} \\
V_{10}, V_{11}: & \bar{x} y \succ \bar{x} \bar{y} \succ x \bar{y} \succ x y
\end{array}
$$

All these linear preference relations follow the order $\mathbf{x}>\mathbf{y}$. Hence, $P \in \operatorname{Legal}(\mathbf{x}>\mathbf{y})$.

Take $r_{\mathbf{x}}$ and $r_{\mathbf{y}}$ both equal to the majority rule, together with a tie-breaking mechanism which, in case of a tie between $x$ and $\bar{x}$ (resp. between $y$ and $\bar{y}$ ), elects $x$ (resp. $y$ ). The projection of $P$ on $\mathbf{x}$ is composed of 7 votes for $x$ and 5 for $\bar{x}$, that is, $P_{i}^{\mathbf{x}}$ is equal to $x \succ \bar{x}$ for $1 \leq i \leq 7$ and to $\bar{x} \succ x$ for $8 \leq i \leq 12$. Therefore $x^{*}=r_{\mathbf{x}}\left(P_{1}^{\mathbf{x}}, \ldots, P_{12}^{\mathbf{x}}\right)=x$ : the $\mathbf{x}-$ winner is $x^{*}=x$. Now, the projection of $P$ on $\mathbf{y}$ given $\mathbf{x}=x$ is composed of 7 votes for $y$ and 5 for $\bar{y}$, therefore $y^{*}=y$, and the sequential winner is now obtained by combining the $\mathbf{x}$-winner and the conditional $\mathbf{y}$-winner given $\mathbf{x}=x^{*}=$ $x$, namely $\operatorname{Seq}\left(r_{\mathbf{x}}, r_{\mathbf{y}}\right)(P)=x y$.

In addition to sequential rules, we define sequential correspondences in a similar way: if for each $i, c_{i}$ is a correspondence on $D_{i}$, then $\operatorname{Seq}\left(c_{1}, \ldots, c_{p}\right)(P)$ is the set of all $\left(x_{1}, \ldots, x_{p}\right)$ s.t. $x_{1} \in c_{1}\left(P_{1}^{\mathbf{x}_{1}}, \ldots, P_{N}^{\mathbf{x}_{1}}\right)$, and for all $i \geq 2$, $x_{i} \in c_{i}\left(P_{i}^{\mathbf{x}_{i}} \mid \mathbf{x}_{1}=x_{1}, \ldots, \mathbf{x}_{i-1}=x_{i-1}, . ., P_{N}^{\mathbf{x}_{i} \mid \mathbf{x}_{1}=x_{1}, ., \mathbf{x}_{i-1}=x_{i-1}}\right)$.

It is important to remark that, in order to compute $\operatorname{Seq}\left(r_{1}, \ldots, r_{p}\right)(P)$, we do not need to know the linear preference relations $V_{1}, \ldots, V_{N}$ entirely: everything we need is the local preference relations: for instance, if $I=\{\mathbf{x}, \mathbf{y}\}$ and $G$ contains the only edge $(\mathbf{x}, \mathbf{y})$, then we need first the unconditional linear preference relations on $\mathbf{x}$ and then the linear preference relations on $\mathbf{y}$ conditioned by the value of $\mathbf{x}$. In other words, if we know the conditional preference tables (for all voters) associated with the graph $G$, then we have enough information to determine the sequential winner for this profile, even though some of the preference relations induced from these tables are incomplete. This is expressed more formally by the following fact (see Observation 4 in [11]): let $I=\left\{\mathbf{x}_{1}, \ldots, \mathbf{x}_{p}\right\}, G$ an acyclic graph over $I$, and $P=\left(V_{1}, \ldots, V_{N}\right), P^{\prime}=\left(V_{1}^{\prime}, \ldots, V_{N}^{\prime}\right)$ two complete preference profiles such that for all $i=$ $1, \ldots, N$ we have $V_{i} \sim_{G} V_{i}^{\prime}$. Then, for any collection of local voting rules $\left(r_{1}, \ldots, r_{p}\right)$, we have $\operatorname{Seq}\left(r_{1}, \ldots, r_{p}\right)(P)=$
$\operatorname{Seq}\left(r_{1}, \ldots, r_{p}\right)\left(P^{\prime}\right)$. (A similar result holds for correspondences.) This implies that applying sequential voting to two profiles corresponding to the same collection of CPnets will give the same result.

We may now wonder whether a Condorcet winner (CW), when there exists one, can be computed sequentially. Sequential Condorcet winners (SCW) are defined similarly as for sequential winners for a given rule: the SCW is the sequential combination of "local" Condorcet winners.

Definition 3 Let $O=\mathbf{x}_{1}>\ldots>\mathbf{x}_{p}$ be a linear order on $I$, and $P \in \operatorname{Legal}(O) .\left(x_{1}^{*}, \ldots, x_{p}^{*}\right)$ is a sequential Condorcet winner for $P$ if and only if

$$
\begin{aligned}
& \text { - } \forall x_{1}^{\prime} \in D_{1}, \#\left\{i, x_{1}^{*} \succ_{i}^{\mathbf{x}_{1}} x_{1}^{\prime}\right\}>\frac{N}{2} \text {; } \\
& \text { - for every } k>1 \text { and } \forall x_{k}^{\prime} \in D_{k} \text {, } \\
& \#\left\{i, x_{k}^{*} \succ_{i}^{\mathbf{x}_{k} \mid \mathbf{x}_{1}=x_{1}^{*}, \ldots, \mathbf{x}_{k-1}=x_{k-1}^{*}} x_{k}^{\prime}\right\}>\frac{N}{2} .
\end{aligned}
$$

Clearly, the existence of a SCW is no more guaranteed than that of a CW, and there cannot be more than one SCW. We have the following positive result in [11] (Proposition 3): if $\left(x_{1}^{*}, x_{2}^{*}, \ldots, x_{p}^{*}\right)$ is a Condorcet winner for $P$, then it is a sequential Condorcet winner for $P$. (Note that the converse fails). An important corollary of this result is the following:

Theorem 3.1 If every $r_{i}$ is Condorcet-consistent then $\operatorname{Seq}\left(r_{1}, \ldots, r_{p}\right)$ is Condorcet-consistent.

Therefore, the output of a sequential voting rule will be the Condorcet winner when there exists one, provided that each local rule $r_{i}$ is Condorcet-consistent. This applies in particular to sequential majority on domains composed of binary issues, which was already known in the particular case when all voters have separable preferences (see [10]). This allows us to claim that the restriction to legal profiles (with respect to some order $O$ ) allows for escaping multiple election paradoxes, at least the version of the paradox that deals with Condorcet winners failing to be elected. For the version of the paradox concerned with electing a Condorcet loser, a sequential voting rule will not elect a Condorcet loser, provided that each of its local rules never elects a Concorcet loser:

Theorem 3.2 If there exists $i \leq p$ s.t. $r_{i}$ never elects a Condorcet loser, then Seq $\left(r_{1}, \ldots, r_{p}\right)$ never elects a Condorcet loser.

For sequential majority on multiple referenda, we have a slightly more significant result:

Theorem 3.3 Let $c_{1}, \ldots, c_{p}$ all equal to the majority correspondence on binary domains. For any o-legal profile $P$ and any $\vec{d} \in \operatorname{Seq}\left(c_{1}, \ldots, c_{p}\right)(P)$, there exist $p$ outcomes $\vec{x}_{1}, \ldots \vec{x}_{p} \in X$ such that $\vec{d}$ weakly Condorcet-dominate $\vec{x}_{i}$ for all $i \leq p$.

This bound $p$ is actually tight, see the next example for $p=3$; it can be generalized to $p \geq 3$. 
Example 3.4 Consider three votes

$$
\begin{aligned}
V_{1}: & : 0_{1} 1_{2} 1_{3} \succ 0_{1} 1_{2} 0_{3} \succ 0_{1} 0_{2} 0_{3} \succ 0_{1} 0_{2} 1_{3} \\
& \succ 1_{1} 1_{2} 1_{3} \succ 1_{1} 0_{2} 1_{3} \succ 1_{1} 1_{2} 0_{3} \succ 1_{1} 0_{2} 0_{3} ; \\
V_{2}: & : 1_{1} 0_{2} 1_{3} \succ 1_{1} 0_{2} 0_{3} \succ 0_{1} 0_{2} 0_{3} \succ 0_{1} 0_{2} 1_{3} \\
& \succ 1_{1} 1_{2} 1_{3} \succ 0_{1} 1_{2} 1_{3} \succ 1_{1} 1_{2} 0_{3} \succ 0_{1} 1_{2} 0_{3} ; \\
V_{3}: & : 1_{1} 1_{2} 0_{3} \succ 1_{1} 0_{2} 0_{3} \succ 0_{1} 0_{2} 0_{3} \succ 0_{1} 1_{2} 0_{3} \\
& \succ 1_{1} 1_{2} 1_{3} \succ 1_{1} 0_{2} 1_{3} \succ 0_{1} 0_{2} 1_{3} \succ 0_{1} 1_{2} 1_{3} .
\end{aligned}
$$

Let $P=\left(V_{1}, V_{2}, V_{3}\right)$, then the sequential majority elects $1_{1} 1_{2} 1_{3}$ from $P$, which only weak Condorcet-dominates three candidates $-1_{1} 0_{2} 1_{3}, 1_{1} 1_{2} 0_{3}$, and $0_{1} 1_{2} 1_{3}$.

\section{Properties of sequential voting rules}

We start be recalling a few important properties that voting rules may (or may not) satisfy. A voting rule satisfies

- anonymity if it is unsensitive to any permutation of the voters;

- homogeneity if for any vote $V$ and any $n \in \mathbb{N}, r(V)=$ $r(n V)$.

- neutrality if for any profile $P$ and any permutation $M$ on candidates, $r(M(P))=M(r(P))$.

- monotonicity if for any profiles $P=\left(V_{1}, \ldots, V_{N}\right)$ and $P^{\prime}=\left(V_{1}^{\prime}, \ldots, V_{N}^{\prime}\right)$ s.t. each $V_{i}^{\prime}$ is obtained from $V_{i}$ by raising only $r(P)$, we have $r\left(P^{\prime}\right)=r(P)$.

- consistency if for any two disjoint profiles (that is, given, by two disjoint electorates) $P_{1}, P_{2}$ s.t. $r\left(P_{1}\right)=$ $r\left(P_{2}\right)$, then $r\left(P_{1} \cup P_{2}\right)=r\left(P_{1}\right)=r\left(P_{2}\right)$.

- participation if for any profile $P$ and any vote $V$, $r(P \cup\{V\})>_{V} r(P)$.

- consensus (or efficiency) if for any profile $P=$ $\left(V_{1}, \ldots, V_{N}\right)$, there is no candidate $c$ s.t. $c>_{V_{i}} r(P)$ for all $i \leq N$.

Since sequential voting rules are sequential composition of multiple local rules, we may wonder whether the properties of local rules carry on to their sequential composition, and vice versa. In this paper, we focus on the above properties. We only give results on voting rules, but most of the them can be easily extended to correspondences.

\subsection{From sequential rules to local rules}

Notice that decomposable voting rules are defined over legal profiles, therefore, when we say a decomposable voting rule satisfies a property involving several profiles, it means that it holds for all legal profiles. This applies to neutrality and monotonicity. Indeed, the usual definition of neutrality (and similarly for efficiency) is not directly applicable to sequential voting rules, because permuting two alternatives in a $O$-legal profile may result in a profile that is not $O$-legal. Therefore, the definition that we take is a straightforward generalization of s-neutrality as defined in [2]: a sequential voting rule $\operatorname{Seq}\left(r_{1}, \ldots, r_{p}\right)$ on $\operatorname{Legal}(O)$ is neutral ${ }^{4}$ if for any permutation $M$ and any $O$-legal profile $P$, if $M(P)$ is $O$-legal, then $M\left(\operatorname{Seq}\left(r_{1}, \ldots, r_{p}\right)(P)\right)=$ $\operatorname{Seq}\left(r_{1}, \ldots, r_{p}\right)(M(P))$. Things are similar for monotonicity of sequential voting rules (and we omit the definition).

Theorem 4.1 If $\operatorname{Seq}\left(r_{1}, \ldots, r_{p}\right)$ satisfies anonymity (resp. homogeneity, neutrality, consistency, participation, consensus), then for any $1 \leq i \leq p, r_{i}$ also satisfies anonymity (resp. homogeneity, neutrality, consistency, participation, consensus).

Monotonicity transfers to the last local rule only. This seemingly strange results is mainly caused by our restriction to legal profiles.

Theorem 4.2 If $\operatorname{Seq}\left(r_{1}, \ldots, r_{p}\right)$ satisfies monotonicity, then $r_{p}$ also satisfies monotonicity.

Since the way to obtain a new legal profile $P^{\prime}$ from $P$ by just raising one candidate can only affect the conditional orders on $D_{p}$, we consider now a stronger monotonicity by allowing multiple candidates to be raised simultaneously.

Definition 4.3 A voting rule $r$ is strongly monotonic if for any profile $P$, any $Y \subseteq X$, and any $P^{\prime}$ obtained from $P$ by only raising the candidates in $Y$ while keeping their relative position unchanged, we have $r\left(P^{\prime}\right) \in r(P) \cup Y$.

Let $Y=\{r(P)\}$, we immediately know if $r$ is strongly monotonic, then it is also monotonic. The next theorem shows that strong monotonicity can be transfers to every local rule (note that the definition of strong monotonicity for sequential rules is conditioned by the profile obtained after permutation is $O$-legal, exactly as for monotonicity.

Theorem 4.4 If Seq $\left(r_{1}, \ldots, r_{p}\right)$ satisfies strong monotonicity, then for any $1 \leq i \leq p, r_{i}$ also satisfies strong monotonicity.

\subsection{From local rules to sequential rules}

Then we give results on whether the sequential composition of local rules inherit a given property satisfied by all local rules. Here are the positive results:

Theorem 4.5 If for all $1 \leq i \leq p, r_{i}$ satisfies anonymity (resp. homogeneity, consistency, strong monotonicity), then $\operatorname{Seq}\left(r_{1}, \ldots, r_{p}\right)$ also satisfies anonymity (resp. homogeneity, consistency, strong monotonicity).

\footnotetext{
${ }^{4} \mathrm{We}$ choose to call this property of sequential voting rules neutrality rather than s-neutrality; it is not ambiguous, provided that $O$ is fixed.
} 
The next theorem shows that the converse of Theorem 4.2 also holds.

Theorem 4.6 If $r_{p}$ satisfies monotonicity, then $\operatorname{Seq}\left(r_{1}, \ldots, r_{p}\right)$ also satisfies monotonicity.

On the other hand, three important properties cannot be lifted from local rules to their sequential composition: neutrality, consensus, and participation. In the case of consensus, this was remarked by several authors in the more specific case of multiple referenda with separable preferences: in [13] it is proven that if there are at least three binary issues (or two binary issues and an even number of voters) then the parallel composition of the majority rule is not efficient (although the majority rule is, of course, efficient). We first give the following result, about neutrality and consensus. Part of it is a corollary of Theorems 1 and 2 in [2].

Theorem 4.7 Let $r_{1}, \ldots, r_{p}, p \geq 2$ be plurality rules and $\left|D_{i}\right| \geq 2$ for all $i \leq p$. If there exists $i \leq p$ s.t. $\left|D_{i}\right|>2$, then Seq $\left(r_{1}, \ldots, r_{p}\right)$ does not satisfy neutrality, nor consensus.

The next example shows that participation cannot be lifted from local rules to their sequential composition.

Example 4.8 Let $\mathcal{N}_{1}, \mathfrak{N}_{2}$ be two CP-nets on $\left\{0_{1}, 1_{1}, 2_{1}\right\} \times$ $\left\{0_{2}, 1_{2}\right\}$ s.t. in $\mathcal{N}_{1}$

$0_{1} \succ_{\mathcal{N}_{1}} 1_{1} \succ_{\mathcal{N}_{1}} 2_{1}, 0_{1}: 0_{2} \succ_{\mathfrak{N}_{1}} 1_{2}, 1_{1}: 1_{2} \succ_{\mathcal{N}_{1}} 0_{2}, 2_{1}: 1_{2} \succ_{\mathcal{N}_{1}} 0_{2}$,

in $\mathcal{N}_{2}, \mathbf{x}_{1}$ and $\mathbf{x}_{2}$ are independent, and $1_{1} \succ_{\mathscr{N}_{2}} 2_{1} \succ_{\mathcal{N}_{2}}$ $0_{1}, 0_{2} \succ_{\mathcal{N}_{2}} 1_{2}$.

Clearly $\mathcal{N}_{2} \not \forall 1_{1} 1_{2} \succ 0_{1} 0_{2}$, therefore, there exists a vote $V_{2}$ consistent with $\mathfrak{N}_{2}$, and $0_{1} 0_{2} \succ_{V_{2}} 1_{1} 1_{2}$, for example

$V_{2}: 2_{1} 0_{2} \succ 2_{1} 1_{2} \succ 1_{1} 0_{2} \succ 0_{1} 0_{2} \succ 1_{1} 1_{2} \succ 0_{1} 1_{2}$

Let $r_{1}$ be the scoring rule with score vector $(3,2,0), r_{2}$ be the plurality rule. Obviously both $r_{1}, r_{2}$ satisfy participation. We consider a profile $P=\left(V_{1}, V_{3}\right)$ s.t. $V_{1}$ and $V_{3}$ are consistent with $\mathcal{N}_{1}$. Then $\operatorname{Seq}\left(r_{1}, r_{2}\right)(P)=0_{1} 0_{2}$ and $\operatorname{Seq}\left(r_{1}, r_{2}\right)\left(P \cup\left\{V_{2}\right\}\right)=1_{1} 1_{2}$. But $0_{1} 0_{2} \succ_{V_{2}} 1_{1} 1_{2}$. Hence Seq $\left(r_{1}, r_{2}\right)$ does not satisfy participation.

The following table summarizes the results of this Section.

\begin{tabular}{|c|c|c|}
\hline Criteria & Global to local & Local to global \\
\hline Anonymity & $\mathrm{Y}$ & $\mathrm{Y}$ \\
\hline Homogeneity & $\mathrm{Y}$ & $\mathrm{Y}$ \\
\hline Neutrality & $\mathrm{Y}$ & $\mathrm{N}$ \\
\hline Monotonicity & Only $r_{p}$ & Only $r_{p}$ \\
\hline Consistency & $\mathrm{Y}$ & $\mathrm{Y}$ \\
\hline Participation & $\mathrm{Y}$ & $\mathrm{N}$ \\
\hline Consensus & $\mathrm{Y}$ & $\mathrm{N}$ \\
\hline Strong monotonicity & $\mathrm{Y}$ & $\mathrm{Y}$ \\
\hline
\end{tabular}

\section{Multiple referenda}

In this section, we focus on the case where all issues are binary (i.e., multiple referenda). Clearly, if $\operatorname{Seq}\left(r_{1}, \ldots, r_{p}\right)$ is "reasonable" to some extent to be defined, then each $r_{i}$ should be the majority rule. We give below a characterization of sequential majority that generalizes May's theorem [12] to multi-issue domains. It is more natural to consider the sequential composition of majority rules as a correspondence, namely $\operatorname{Seq}\left(c_{1}, \ldots, c_{p}\right)$, where each $c_{i}$ is the majority correspondence for two candidates. Notice if the number of voters is odd, then sequential majority outputs a single winner, which obviously is not necessarily the case where the number of voters is even: for instance, let us consider 2 voters, with respective preference orders $x y \succ x \bar{y} \succ \bar{x} y \succ \bar{x} \bar{y}$ and $\bar{x} y \succ \bar{x} \bar{y} \succ x \bar{y} \succ x y$. The profile is legal for $\mathbf{x}>\mathbf{y}$, and the outcome of sequential majority consists here of the set of three alternatives $\{x y, x \bar{y}, \bar{x} y\}$.

First we make an observation on the neutrality of each $c_{i}$. Our aim is to find a necessary and sufficient condition for each $c_{i}$ to be neutral, based on some observations on $\operatorname{Seq}\left(c_{1}, \ldots, c_{p}\right)$. Recall that in Theorem 4.1 it has been proved that if $\operatorname{Seq}\left(c_{1}, \ldots, c_{p}\right)$ is neutral then $c_{i}$ is neutral. But this is not a necessary condition (and we will prove that if $p \geq 3$ then the sequential majority is not neutral, see Theorem 5.8). Fortunately, for multiple referenda, we can find a suitable condition. Denote $M_{R}$ the permutation on $x$ that exchanges $\left(d_{1}, \ldots, d_{p}\right)$ to $\left(\overline{d_{1}}, \ldots, \overline{d_{p}}\right)$, for example $M_{R}\left(0_{1} 1_{2} 0_{3}\right)=1_{1} 0_{2} 1_{3}$. We say that $\operatorname{Seq}\left(c_{1}, \ldots, c_{p}\right)$ is insensitive to $M_{R}$ if for any legal profile $P, M_{R}\left(\operatorname{Seq}\left(c_{1}, \ldots, c_{p}\right)(P)\right)=\operatorname{Seq}\left(c_{1}, \ldots, c_{p}\right)\left(M_{R}(P)\right)$. The next theorem says that a decomposable voting correspondence is insensitive to $M_{R}$ iff its local correspondences are neutral.

Theorem $5.1 c_{i}$ is neutral for all $i \leq p$ if and only if $\operatorname{Seq}\left(c_{1}, \ldots, c_{p}\right)$ is insensitive to $M_{R}$.

The next theorem characterizes sequential composition of majority correspondences.

\section{Theorem 5.2}

1. On the domain of all profiles that consists of odd number of votes, a decomposable voting correspondence $\operatorname{Seq}\left(c_{1}, \ldots, c_{p}\right)$ is the sequential majority correspondence if and only if $\operatorname{Seq}\left(c_{1}, \ldots, c_{p}\right)$ satisfies anonymity, strong monotonicity, and is insensitive to $M_{R}$.

2. A decomposable correspondence $C=\operatorname{Seq}\left(c_{1}, \ldots, c_{p}\right)$ is the sequential majority correspondence if and only if it satisfies anonymity, strong monotonicity, consistency, and insensitivity to $M_{R}$, and if whenever $|C(P)| \geq 2$ for some profile $P$, then $|P|$ is even.

Remark that the sets of properties in 1. and 2. are minimal (for instance, in 1., all three properties are required). To see the property set in 2 . is minimal, we present examples for removing each condition. Anonymity is obvious.

- For strong monotonicity, let each $c_{i}$ be the correspondence that select a minority. Then $c_{i}$ is consistent and neu- 
tral, so $C$ satisfies consistency by Theorem $4.5, C$ is insensitive to $M_{R}$ by Theorem 5.1. Clearly when $|P|$ is odd, $|C(P)|=1$. So $C$ satisfies the other four conditions, and is not the sequential composition of majority correspondences.

-For consistency, let $c_{i}$ be majority correspondence if $|P|$ is odd, otherwise it is trivial (always outputs $D_{i}$ ). Since majority and trivial correspondence are both neutral and strong monotonic, we know $C$ satisfies strong monotonicity and is insensitive to $M_{R}$ by Theorem 4.5. Notice when $|P|$ is odd, $C$ is the sequential composition of majority correspondence, we know $C$ satisfies the four properties other than consistency.

-For insensitiveness to $M_{R}$, we simply let $c_{i}(P)=0_{i}$ for all $P$, it is easy to check $C$ satisfies other four properties.

-For $|C(P)| \geq 2 \Rightarrow|P|$ is odd, we consider the trivial correspondence $C(P)=X$ for all $P$. By simple calculation we know all the other four properties holds.

So the property set in 2 . is minimal. Similar examples show the property set in 1 . is also minimal.

Recall that Theorem 4.7 says that if some $\left|D_{i}\right|>2$, then the sequential composition of local rules satisfying neutrality (resp. consensus) might not satisfy neutrality (resp. consensus). We may wonder how about if $\left|D_{i}\right|=2$ for all $i$. Notice first that plurality and majority coincides on binary domains. We observe that when $p=2$, sequential majority is neutral.

Theorem 5.3 Let $c_{1}, c_{2}$ be equal to the majority correspondence on binary domains. Then $\operatorname{Seq}\left(c_{1}, c_{2}\right)$ is a neutral correspondence.

Then we are interested in $p \geq 2$. In [2], two theorems were provided to characterize the neutrality and consensus (i.e. Pareto optimality) of voting rules over separable profiles.

Theorem 5.4 (Theorem 1 in [2]) Over separable profiles, if at least one of the following two conditions holds

$$
\begin{aligned}
& \text { 1. }|p \geq 3| \text { and }\left|D_{i}\right| \geq 2 \text { for all } i \leq p \text {, or } \\
& \text { 2. } p=2,\left|D_{1}\right| \geq 2,\left|D_{2}\right| \geq 2 \text {, and }\left|D_{1}\right| \geq 3 \text { or }\left|D_{2}\right| \geq 3 \text {. }
\end{aligned}
$$

then the only sequential voting rule satisfying consensus is the dictatorship,

Theorem 5.5 (Theorem 2 in [2]) Suppose $p \geq 2,\left|D_{i}\right| \geq 2$ and $r_{i}$ satisfies consensus for all $i \leq p$, if $\operatorname{Seq}\left(r_{1}, \ldots, r_{p}\right)$ is neutral over the set of all separable profiles, then it must be a dictatorship.

It is easy to check that if we extend the domain of the rules to all $O$-legal profiles, the theorems still hold. This observation sheds some light on decomposable voting rules satisfying neutrality or consensus, as the following corollaries show.
Corollary 5.6 Scoring rules, Bucklin, Maximin, Copeland and Ranked pairs are not decomposable over combinatorial domains other than $p=2$ and $\left|D_{1}\right|=\left|D_{2}\right|=2$.

Corollary 5.7 If $p \geq 3$, then the sequential composition of plurality on p binary domains does not satisfy neutrality, nor consensus.

This corollary together with Theorem 4.7 and Theorem 5.3 tells us that the only neutral sequential plurality rule is the one on a $2 \times 2$ domain.

Theorem 5.8 A sequential composition of plurality rule is neutral iff $p=2$ and $\left|D_{1}\right|=\left|D_{2}\right|=2$.

We can also prove that for $p \geq 3$, if a decomposable voting rule satisfies neutrality, then it is not Condorcet-consistent. Notice that since a Condorcet-consistent rule does not necessarily satisfy consensus, this result is not a corollary of Theorem 5.4 or Theorem 5.5. Since this paper mainly discusses multiple referenda, we do not give this result formally.

Remember that in Theorem 5.5, each $r_{i}$ satisfying consensus is required for the dictatorship. We may wonder what we can learn if we assume only the neutrality of $\operatorname{Seq}\left(r_{1}, \ldots, r_{p}\right)$. The next theorem says that for multiple referenda, if a voting rule satisfies decomposability and neutrality, then the winner of a profile, or its complement, should be ranked first by at least one of the voters. Here the complement $\bar{x}=\left(\bar{d}_{1}, \ldots, \bar{d}_{p}\right)$ of $x=\left(d_{1}, \ldots, d_{p}\right)$ means that $\bar{d}_{j} \neq d_{j}$ for all $j \leq p$. Notice that we are talking about multiple referenda where $\left|D_{i}\right|=2, \bar{x}$ is uniquely determined.

Theorem 5.9 If a sequential voting rule $\operatorname{Seq}\left(r_{1}, \ldots, r_{p}\right)$ on a domain consisting of binary issues satisfies neutrality, then for any preference profile $P=\left\{V_{1}, \ldots, V_{N}\right\}$ following $O, \vec{x}=\operatorname{Seq}\left(r_{1}, \ldots, r_{p}\right)(P)$ or $\overline{\vec{x}}$ must be top ranked in at least one of $\left\{V_{1}, \ldots, V_{N}\right\}$.

We end this section with some considerations on manipulability. We know that the majority rule for 2 candidates is not manipulable. What about sequential majority? We know from [10] that if all voters have separable preferences, then sequential majority is non-manipulable. Does this extend to legal profiles in which some voters have nonseparable preferences? Unfortunately, it does not:

\section{Theorem 5.10 Sequential majority is manipulable.}

This is easily seen on this counterexample with two binary issues $\mathbf{x}$ and $\mathbf{y}$ : voter 1 has the preference relation $x y \succ \bar{x} y \succ$ $x \bar{y} \succ \bar{x} \bar{y}$, voter 2 has $x \bar{y} \succ x y \succ \bar{x} y \succ \bar{x} \bar{y}$ and voter 3 has $\bar{x} y \succ$ $\bar{x} \bar{y} \succ x \bar{y}>x y$. The profile is in Legal $(\mathbf{x}>\mathbf{y})$. If 1 knows the preferences of 2 and 3 then he has no interest to vote sincerely on issue $\mathbf{x}$, even though his preference relation is separable: if he votes sincerely, then he votes $x$ and then the outcome is $x \bar{y}$. If he votes for $\bar{x}$ instead, then the outcome is $\bar{x} y$, which is better to him. 
As a corollary of this result, strategyproofness does not transfer from the local level to the global level.

\section{Discussion}

We have shown that the sequential composition of local voting rules allows for escaping usual multiple election paradoxes, under a domain restriction much weaker than separability. Moreover, these sequential rules have a cheap communication complexity. We have established many results concerning the transfer (or the failure of transfer) of important properties from local rules to/from their sequential composition.

Interestingly, our work has benefited from several previous streams of work that were almost unrelated: on the one hand, social choice, and on the other hand, conditional preferential independence, initially developed in the literature of multiattribute decision making and now widely used in artificial intelligence (with CP-nets). The initial motivation of our work was also inspired by the notion of cheap protocol, as defined in the literature on communication complexity.

An important aspect of multiple election paradoxes that would deserve more attention is the role of knowledge. What makes our protocols interesting is the conjunction of two properties: they are cheap (in terms of communication complexity) and epistemically safe: our domain restriction ensures that each time an elicitation query is asked to the voters, the voters know the answer, that is, they have all the necessary information needed to give the answer. Multiple election paradoxes, where voters experience regret after voting for a given issue when learning the outcome of other issues, is to a large extent due to the fact that voters are asked to cast a vote about a given issue whereas they don't know their true preference, the latter depending on the value of some other issues. This, of course, is guaranteed with separability, but this assumption is far too demanding. We believe that our restriction to legal profiles constitutes a reasonable sufficient condition for the existence of a cheap and epistemically safe protocol. However it is not necessary, because we may consider sequential rules where the order in which the issues are considered depends on the value of some previously decided issue; these rules would work for a more general class of profiles. Looking for a sufficient and necessary condition is left for further study, as well as a formalization of epistemically safe protocols within epistemic logic.

\section{Acknowledgements}

This work was partly supported by the National Foundation of Natural Sciences of China (Grant No: 60621062, 60496321), and by the French ANR project "PHAC" (Preference Handling and Aggregation over Combinatorial Domains).

\section{References}

[1] J.-P. Benoit and L.A. Kornhauser. Voting simply in the election of assemblies. Technical Report 91-32, C.V. Starr Center for Applied Economics, 1991.

[2] J.-P. Benoit and L.A. Kornhauser. Only a dictatorship is efficient or neutral. Technical report, 2006.

[3] C. Boutilier, R. Brafman, C. Domshlak, H. Hoos, and D. Poole. CP-nets: a tool for representing and reasoning with conditional ceteris paribus statements. Journal of Artificial Intelligence Research, 21:135-191, 2004.

[4] S. Brams and P. Fishburn. Voting procedures. In K. Arrow, A. Sen, and K. Suzumura, editors, Handbook of Social Choice and Welfare, chapter 4. Elsevier, 2004.

[5] S. Brams, D. Kilgour, and W. Zwicker. Voting on referenda: the separability problem and possible solutions. Electoral Studies, 16(3):359-377, 1997.

[6] S. Brams, D. Kilgour, and W. Zwicker. The paradox of multiple elections. Social Choice and Welfare, 15(2):211-236, 1998.

[7] V. Conitzer and T. Sandholm. Communication complexity of common voting rules. In Proceedings of the 6th ACM Conference on Electronic Commerce (EC-05), pages 78-87, 2005.

[8] J. Hodge. Separable preference orders. PhD thesis, Western Michigan University, 2002.

[9] R. Keeney and H. Raiffa. Decision with Multiple Objectives: Preferences and Value Tradeoffs. Wiley and Sons, 1976.

[10] D. Lacy and E. Niou. A problem with referenda. Journal of Theoretical Politics, 12(1):5-31, 2000.

[11] J. Lang. Voting and aggregation on combinatorial domains with structured preferences. In Proceedings of the Twenthieth Joint International Conference on Artificial Intelligence (IJCAI'07), 2007.

[12] K. May. A set of independent necessary and sufficient conditions for simple majority decisions. Econometrica, 20:680-684, 1952.

[13] İ. Özkal-Sanver and R. Sanver. Ensuring Pareto-optimality by referendum voting. Social Choice and Welfare, 27:211219, 2006.

[14] C. Plott and M. Levine. A model of agenda influence on committee decisions. The American Economic Review, 68(1):146-160, 1978.

[15] M. Scarsini. A strong paradox of multiple elections. Social Choice and Welfare, 15(2):237-238, 1998. 


\section{Appendix 1: conditional preference networks (CP-nets)}

Let $I$ ba a finite set of variables, and for each $\mathbf{x}_{i} \in I$, let $D_{i}$ be a finite value domain. Let $x=\prod_{\mathbf{x}_{i} \in I} D_{i}$.

A CP-net over $I$ is a pair $\mathcal{N}=\langle G, C P T\rangle$, where $G$ is a directed graph over $I$ and $C P T$ is a set of conditional preference tables $\left\{C P T\left(\mathbf{x}_{i}\right): \mathbf{x}_{i} \in I\right\}$. Each conditional preference table $C P T\left(\mathbf{x}_{i}\right)$ associates a total order $\succ_{\vec{u}}^{i}$ over $D_{i}$, with each instantiation $\vec{u}$ of $\mathbf{x}_{i}$ 's parents $\operatorname{Par}\left(\mathbf{x}_{i}\right)=U$, where $\operatorname{Par}\left(\mathbf{x}_{i}\right)$ denote the parents of $\mathbf{x}_{i}$ in $G$.

For instance, let $I=\{\mathbf{x}, \mathbf{y}, \mathbf{z}\}$, all three being binary, and assume that preference of a given agent over $2^{I}$ can be defined by a CP-net whose structural part is the directed acyclic graph $G=\{(x, y),(y, z),(x, z)\}$; this means that the agent's preference over the values of $\mathbf{x}$ is unconditional, preference over the values of $\mathbf{y}$ (resp. $\mathbf{z}$ ) is fully determined given the value of $\mathbf{x}$ (resp. the values of $\mathbf{x}$ and $\mathbf{y}$ ).

Example 5 Let $N=12, I=\{\mathbf{x}, \mathbf{y}\}$ with $\operatorname{Dom}(\mathbf{x})=\{x, \bar{x}\}$ and $\operatorname{Dom}(\mathbf{y})=\{y, \bar{y}\}$, and $P=\left(V_{1}, \ldots, V_{12}\right)$ the following 12-voter profile:

$$
\begin{array}{ll}
V_{1}, V_{2}, V_{3}, V_{4}: & x y \succ \bar{x} y \succ x \bar{y} \succ \bar{x} \bar{y} \\
V_{5}, V_{6}, V_{7}: & x \bar{y} \succ x y \succ \bar{x} y \succ \bar{x} \bar{y} \\
V_{8}, V_{9}, V_{10}: & \bar{x} y \succ \bar{x} \bar{y} \succ x y \succ x \bar{y} \\
V_{11}, V_{12}: & \bar{x} y \succ \bar{x} \bar{y} \succ x \bar{y} \succ x y
\end{array}
$$

All these linear preference relations are compatible with the graph $G$ over $\{\mathbf{x}, \mathbf{y}\}$ whose single edge is $(\mathbf{x}, \mathbf{y})$; equivalently, they follow the order $\mathbf{x}>\mathbf{y}$ : for all voters, the preference on $\mathbf{x}$ is

\begin{tabular}{|c|c|c|c|}
\hline oters $1,2,3,4$ & voters $5,6,7$ & voters $8,9,10$ & voters 11,12 \\
\hline$x \succ$ & $x \succ$ & $\bar{x} \succ x$ & $\bar{x} \succ x$ \\
\hline$x: y \succ \bar{y}$ & $x: \bar{y} \succ y$ & $x: y \succ \bar{y}$ & $x: \bar{y} \succ y$ \\
\hline $\bar{x}: y \succ \bar{y}$ & $\bar{x}: y \succ \bar{y}$ & $\bar{x}: y \succ \bar{y}$ & $\bar{x}: y \succ \bar{y}$ \\
\hline
\end{tabular}
unconditional and the preference on $\mathbf{y}$ may depend on the value of $\mathbf{x}$.

The corresponding conditional preference tables are:

The conditional preference statements contained in these tables are written with the following usual notation: for instance, in a CP-net $\mathcal{N}, x_{1} \overline{x_{2}}: x_{3} \succ \overline{x_{3}}$ means that when $\mathbf{x}_{1}$ is true and $\mathbf{x}_{2}$ is false then $\mathbf{x}_{3}=x_{3}$ is preferred to $\mathbf{x}_{3}=\overline{x_{3}}$ ceteris paribus, that is, for any fixed values of the other variables $\mathbf{x}_{4}, \ldots, x_{p}$.

Formally in CP-net $\mathcal{N}$, for any $\mathbf{x}_{i} \in I$, the conditional independence in CP-net leads to the following preference relations. Define first

$$
\succ^{\mathbf{x}_{i}}=\left\{\vec{u} \vec{z} x \succ_{\mathcal{N}} \vec{u} \vec{z} y: x \succ_{\vec{u}}^{i} y, \vec{z} \in \prod_{\mathbf{x}_{j} \notin \operatorname{Par}\left(\mathbf{x}_{i}\right)} D_{j}\right\} .
$$

Write $\succ^{\mathcal{N}}=\bigcup_{\mathbf{x}_{i}} \succ^{\mathbf{x}_{i}}$ the union of all relations $\succ^{\mathbf{x}_{i}}$ encoded in $\operatorname{CPT}\left(\mathbf{x}_{i}\right)$. Notice we require $\succ_{\mathcal{X}}$ be a linear order, so $\succ_{\mathcal{X}}$ is transitive. Therefore the full preferential information encoded in $\mathcal{N}$ is the transitive closure of $\succ^{\mathcal{X}}$, namely $\succ_{\mathcal{N}}=\overline{\succ^{\mathcal{X}}}$. It has been proved [3] that if $G$ is acyclic, then $\succ_{\mathcal{N}}$ is consistent, namely for any $\vec{x}, \vec{y}$, at most one of $\vec{x} \succ_{\mathscr{N}} \vec{y}$ and $\vec{y} \succ_{\mathscr{N}} \vec{x}$ holds.
In the paper we make the classical assumption that $G$ is acyclic. A CP-net $\mathcal{N}$ induces a preference ranking on $x: \mathcal{N}=\vec{x} \succ \vec{y}$ iff $\vec{x} \succ_{\mathcal{X}} \vec{y}$. Notice for any $\vec{x} \succ^{\mathcal{X}} \vec{y}, \vec{x}$ and $\vec{y}$ differs only in one issue, and $\vec{z} \succ_{\mathcal{X}} \vec{w}$ is obtained through a transitive sequence of relations $\vec{z} \succ^{\mathcal{N}} \vec{x}_{1}, \vec{x}_{1} \succ^{\mathcal{N}} \vec{x}_{2}, \ldots, \vec{x}_{m-1} \succ^{\mathcal{N}} \vec{x}_{m}, \vec{x}_{m} \succ^{\mathcal{N}} \vec{w}$. So $\mathcal{N}=\vec{x} \succ$ $\vec{y}$ is thus equivalent to: There is a sequence of improving flips from $\vec{y}$ to $\vec{x}$, where an improving flip is the flip of a single issue $\vec{x}_{i}$ "respecting" the preference table $C P T\left(\mathbf{x}_{i}\right)$ (see [3]). Note that the preference relation induced from a CP-net is generally not complete, as seen on the following example.

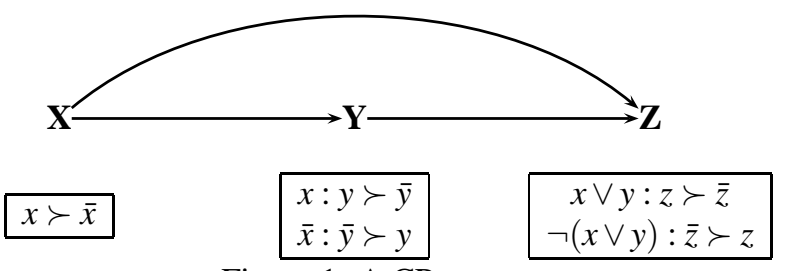

Figure 1: A CP-net.

Example 6 Consider the example depicted in Figure 1.

$\succ^{\mathbf{x}}: \quad x y z \succ \bar{x} y z, \quad x y \bar{z} \succ \bar{x} y \bar{z}, x \bar{y} z \succ \bar{x} \bar{y} z, x \bar{y} \bar{z} \succ \bar{x} \bar{y} \bar{z}$

$\succ^{\mathbf{y}}: x y z \succ x \bar{y} z, \quad x y \bar{z} \succ x \bar{y} \bar{z}, \quad \bar{x} \bar{y} z \succ \bar{x} y z, \quad \bar{x} \bar{y} \bar{z} \succ \bar{x} y \bar{z}$

$\succ^{\mathbf{z}}: x y z \succ x y \bar{z}, x \bar{y} z \succ x \bar{y} \bar{z}, \bar{x} y z \succ \bar{x} \bar{y} z, \bar{x} \bar{y} \bar{z} \succ \bar{x} \bar{y} z$, illustrated as

Now, $\succ_{\mathcal{N}}$ is the transitive closure of $\succ^{\mathbf{x}} \cup \succ^{\mathbf{y}} \cup \succ^{\mathbf{z}}$, illustrated by the following diagram:

$$
x y z \searrow_{x y \bar{z}}^{x \bar{y} z} \searrow^{\searrow} x \bar{y} \bar{z} \rightarrow \bar{x} \bar{y} \bar{z} \rightarrow \bar{x} \bar{y} z \rightarrow \bar{x} y z \rightarrow \bar{x} y \bar{z}
$$

To see how to generate $x y z \succ \bar{x} \bar{y} \bar{z}$, we consider a three-step increasing flip: in the first step, $\mathbf{x}$ is flipped according to $x \succ \bar{x}$, thus $x \bar{y} \bar{z}$ is obtained; then in the second step, $\mathbf{z}$ is flipped according to $x \bar{y}: z \succ \bar{z}$, thus leads to $x \bar{y} z$; finally $\mathbf{y}$ is flipped according to $x: y \succ \bar{y}$, reaching $x y z$.

An important property of such sequential voting rules and correspondences is that the outcome does not depend on $O$, provided that $G$ follows $O$. This can be expressed formally:

Observation 1 Let $O=\left(\mathbf{x}_{1}>\ldots>\mathbf{x}_{p}\right)$ and $O^{\prime}=\left(\mathbf{x}_{\sigma(1)}>\ldots>\right.$ $\left.\mathbf{x}_{\sigma(p)}\right)$ be two linear orders on $V$ such that $G$ follows both $O$ and $O^{\prime}$. Then

$$
\operatorname{Seq}\left(r_{1}, \ldots, r_{p}\right)(P)=\operatorname{Seq}\left(r_{\sigma(1)}, \ldots, r_{\sigma(p)}\right)(P)
$$

and similarly for voting correspondences.

Example 7 Everything is as in Example 5, except that we don't know the voters' complete preference relations, but only their corresponding conditional preference tables. These conditional preferences contain strictly less information than $P$, because some of the preference relations they induce are not complete: for instance, the induced preference relation for the first 4 voters is $x y \succ \bar{x} y \succ \bar{x} \bar{y}, x y \succ x \bar{y} \succ \bar{x} \bar{y}$, with $x \bar{y}$ and $\bar{x} y$ being incomparable. However, we have enough information to determine the sequential winner for this profile, even though some of the preference relations are incomplete. For instance, taking again the majority rule for $r_{\mathbf{x}}$ and $r_{\mathbf{y}}$, the sequential winner is xy for any complete profile $P^{\prime}=\left(V_{1}^{\prime}, \ldots, V_{12}^{\prime}\right)$ extending the incomplete preference relations induced by the 12 conditional preference tables above. 